This item was submitted to Loughborough's Research Repository by the author.

Items in Figshare are protected by copyright, with all rights reserved, unless otherwise indicated.

\title{
Smarter maintenance through internet-based condition monitoring with indirect sensing, novelty detection, and $\mathrm{XML}$
}

PLEASE CITE THE PUBLISHED VERSION

PUBLISHER

Professional Engineering Publishing / @ IMECHE

VERSION

VoR (Version of Record)

\section{LICENCE}

CC BY-NC-ND 4.0

\section{REPOSITORY RECORD}

Zorriassatine, F., Bilal Ashraf, L. Notini, Robert M. Parkin, Michael R. Jackson, and J. Coy. 2019. "Smarter Maintenance Through Internet-based Condition Monitoring with Indirect Sensing, Novelty Detection, and XML". figshare. https://hdl.handle.net/2134/4736. 
This item was submitted to Loughborough's Institutional Repository (https://dspace.lboro.ac.uk/) by the author and is made available under the following Creative Commons Licence conditions.

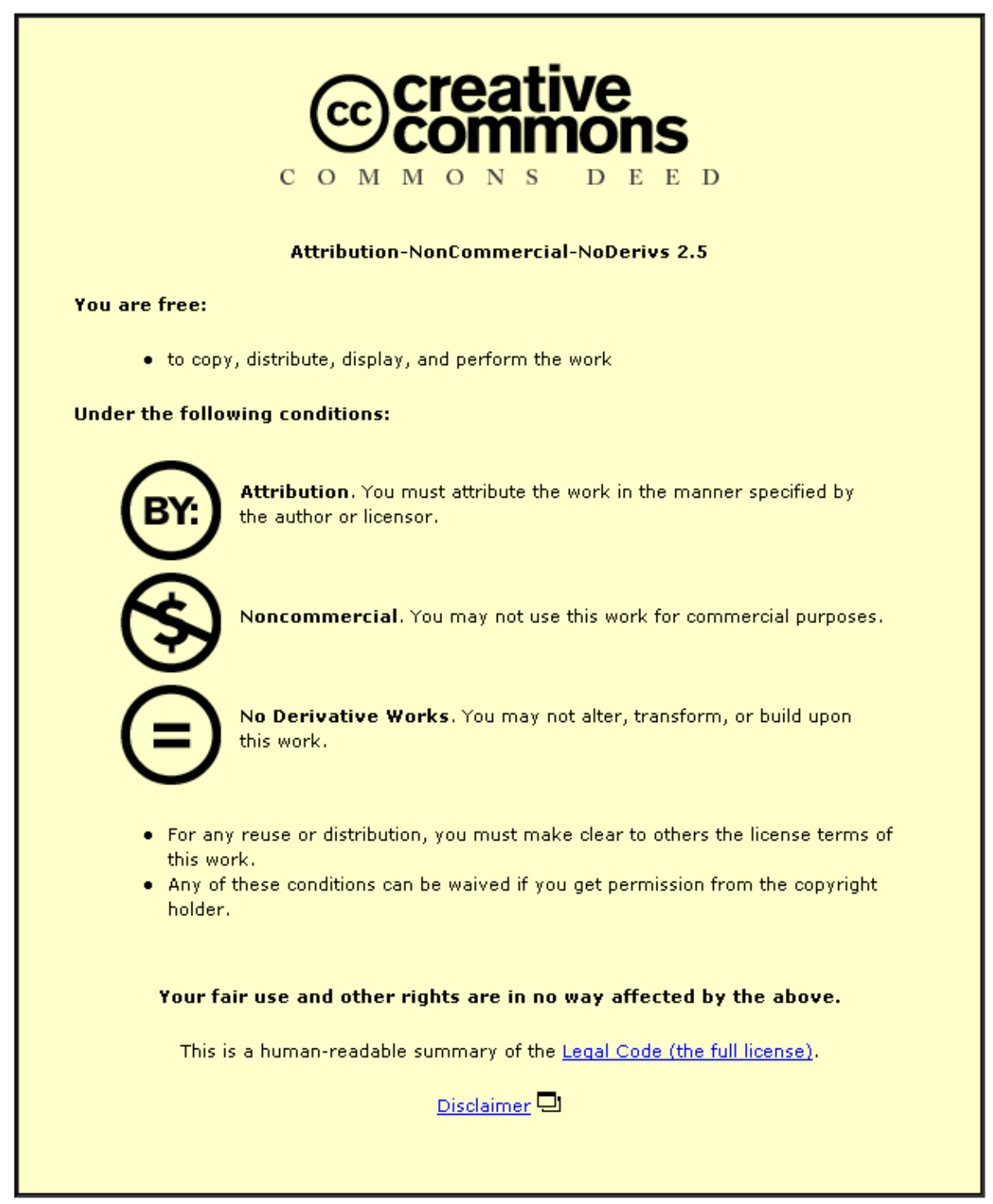

For the full text of this licence, please go to: http://creativecommons.org/licenses/by-nc-nd/2.5/ 


\title{
Smarter maintenance through internet-based condition monitoring with indirect sensing, novelty detection, and XML
}

\author{
F Zorriassatine $^{1 *}$, B Ashraf ${ }^{1}$, L Notini $^{1}$, R M Parkin ${ }^{1}$, M R Jackson ${ }^{1}$, and J Coy ${ }^{2}$ \\ ${ }^{1}$ Mechatronics Research Centre, Loughborough University, Loughborough, UK \\ ${ }^{2}$ Technology Centre, Royal Mail Group plc, Swindon, UK
}

The manuscript was received on 26 August 2004 and was accepted after revision for publication on 10 March 2005.

DOI: 10.1243/095965105X9605

\begin{abstract}
In engineering, combining a number of solutions and technologies can result in more effective systems than using only one approach on its own. In particular, it has been shown that in condition monitoring (CM), smarter maintenance systems may be obtained by integrating various sensors together. This paper extends this idea by integrating various non-homogeneous technologies horizontally. The proposed system is an internet-based condition monitoring (e-CM) prototype that can identify abnormal tension in moving belts. It is shown that by applying a classification technique, known as novelty detection, it is possible to decide the status of belt tension by processing the belt vibration signals from an optical sensor (i.e. an indirect sensing approach). A novel method for industrial network communication using XML to create a single standard format for sensor information is also used to link the sensor to the process controller via the internet using the flexible CAN bus technology; this is used together with low-cost microcontrollers with a built-in ethernet link for data acquisition and transmission. The resulting integrated approach is more efficient because: (a) it can reduce waste by minimizing process interruptions caused by direct belt inspection methods while obtaining high detection accuracy (99.67 per cent) and (b) it can provide on-line remote CM that is cost-effective, simple, standardized, and scalable across a wide area and for a relatively large number of sensors. This improvement is especially important when applied to bottleneck processes and critical components.
\end{abstract}

Keywords: condition monitoring, indirect sensing, novelty detection, belt, XML

\section{INTRODUCTION}

The fierce competition to gain secure and stable market positions is the driving force behind the move for lower costs, faster and more stringent control, and minimization of human operator involvement. This phenomenon is ever more present in all types of industry, including manufacturing as well as the service sector.

Royal Mail, which is one of the largest service providers in the United Kingdom, is no exception to the above observation. The importance of prompt delivery of mail items (letters and parcels alike) dictates that all operations including the automated mail sorting

\footnotetext{
* Corresponding author: Mechatronics Research Centre, Wolfson School of Mechanical and Manufacturing Engineering, Loughborough University, Loughborough, Leicestershire LE11 3UT, UK. email: zorsat@yahoo.co.uk
}

plants maintain reliable and uninterrupted process runs. This is especially true during the peak times when the volume of mail to be processed is so high that process delays can result in severe delivery delays, increased mail backlogs, and associated redistribution and reprocessing costs, and occasionally high fines imposable by the government-appointed regulating bodies.

In most of the mail sorting centres (a total of 75 such centres throughout the UK) there are a number of different types of automated mail processing machinery. In particular, one group of these machines, i.e. the integrated mail processing (IMP) units, can process up to 40000 letters per hour each. Typically, most mail centres have two or three IMPs, each of which consists of approximately 700 rolling element bearings and 300 conveyor belts of different sizes and specifications (e.g. see Fig. 1). The importance of reliable belts and bearings operation is even easier 


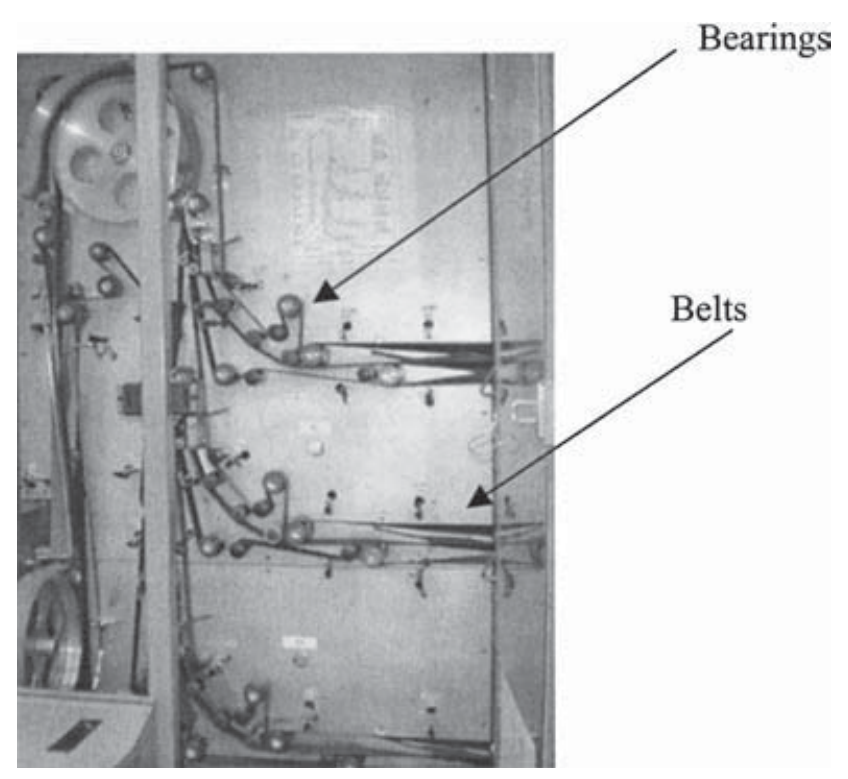

Fig. 1 Extensive deployment of rolling element bearings and belts for transporting mail items within the processing machinery

to understand if the above component figures are scaled up to cover the entire set of mail sorting plants nationally in the UK.

A belt-based motion transmission system possesses a number of possible failure modes. These include wear or contamination of the surface of the belt (leading to slippage and reduced power transmission capacity), tension layer faults (such as non-uniformity of elastic modulus and non-uniformity of tension distribution), the embedment or adhesion of an object on the belt surfaces, and pulley misalignment.

Previous studies of belt faults have shown that belt failure modes affect the characteristics of belt vibration. It has been shown that changes in the Fourier decomposition of belt vibration occur in response to an increase in slippage [1]. Belt joints and other forms of tension layer non-uniformity affect characteristics of belt vibration [2-4]. In reference [5] the focus of study consists of the effect on the characteristics of belt vibration of the presence of an object, in the form of a weld, on the layer of a metal belt that contacts the system pulleys. It has been shown that all the failure modes mentioned affected vibration on a pulley mounting [6]. Since coupling mechanisms between pulley mountings and belts exist, then the work referred to also provides additional evidence that various belt fault modes affect belt vibration.

Condition monitoring (CM) diagnoses faults by continuously obtaining and analysing signals from a process. $\mathrm{CM}$, in general, has been identified as an effective maintenance strategy for Royal Mail's automated mail processing plants as it can provide machine fault information, either in advance or before a machine or its components reach critical conditions, in order to allow timely and appropriate maintenance actions to be taken [7]. In particular, efficient and effective monitoring of the moving belts is one of the several vital routes of implementing a CM strategy at Royal Mail. This study focuses on the $\mathrm{CM}$ of belts with respect to their tension variations.

Engineering and science are evolving in all directions. As in most areas of engineering, new techniques for condition monitoring are emerging and it is difficult to keep abreast of all these developments. Many agencies have been active in committing personnel and financial resources in programmes to develop better and smarter diagnostic systems in order to monitor process deterioration and predict its failure [8]. Combining a number of solutions and technologies can often result in more effective systems compared with situations when only one approach is used on its own in order to improve an existing system. In particular, it has been shown that in CM, smarter maintenance systems may be obtained by integrating various sensors together (see, for example references [9] and [10]). This paper extends this idea by integrating various nonhomogeneous technologies horizontally in order to achieve smarter CM.

It is shown here that by intelligently combining a number of different techniques, e.g. novelty detection for data modelling, with some of the evolving technologies such as the internet, together with the existing condition monitoring methods (e.g. belt vibration measurement), a smarter maintenance system can be obtained. The resulting system will be more efficient and flexible than the ones obtained through the prevailingly isolated introduction of new approaches to $\mathrm{CM}$.

\section{DETECTING CHANGES IN BELT TENSION}

\subsection{Belt tension measurements}

The tension in the spans of a stationary belt (known as 'the initial tension') is an important factor when designing and maintaining belt drives. If the initial tension is too high then components connected to the drive may be damaged due to exposure to excessive stress [11]. If the initial tension is too low then this limits the maximum power that can be transmitted by a belt drive, which may lead to the occurrence of excessive slippage [12]. Slippage accelerates belt surface wear and also affects the 
energy efficiency of the belt drive. In order to execute the condition-based maintenance of belt drives, it is necessary that non-negligible changes in belt-drive variables, such as the initial tension, are detectable. Once such changes are detectable maintenance tasks can be scheduled accordingly.

It has been shown that characteristics of the transverse vibration of a belt drive are affected by various variables $[2, \mathbf{1 3}]$. One such characteristic is the resonance frequency of a belt drive, which is dependent on initial belt tension (among other variables). This is the case in both stationary belt spans and belt spans of a drive that is transmitting mechanical power.

Various methods have been developed to assist in both the assembly and maintenance of belt drives. For example, a device for measuring the resonance frequency of a stationary belt [14] was developed in order to facilitate the accurate measurement of the initial belt tension. This method requires that the resonance frequency of a belt is excited. Excitation of the resonance frequency of a belt span may be achieved by subjecting the span to an approximation of a unit impulse. The unit impulse approximation is generated through a collision between the span and an object, such as the reciprocating element of a solenoid. When a belt is in motion transverse vibration of the belt spans is produced through mechanisms such as parametric excitation [15].

\subsection{Collecting transverse vibration signals}

The following equations constitute the basic equations of motion for a string coupling two fixed pulleys (Fig. 2) and travelling at a constant velocity [16]

$$
\begin{gathered}
\frac{\mathrm{d}^{2} y}{\mathrm{~d} t^{2}}=c^{2} \frac{\mathrm{d}^{2} y}{\mathrm{~d} x^{2}}-v^{2} \frac{\mathrm{d}^{2} y}{\mathrm{~d} x^{2}}-2 v \frac{\mathrm{d}^{2} y}{\mathrm{~d} x \mathrm{~d} t} \\
-\Gamma\left(\frac{\mathrm{d} y}{\mathrm{~d} t}+v \frac{\mathrm{d} y}{\mathrm{~d} x}\right)+\frac{\mathrm{d}^{4} y}{\mathrm{~d} x^{4}} \\
c=\left(\frac{T_{0}}{m}\right)^{1 / 2}
\end{gathered}
$$

The right-hand side of the equation (1) includes (respectively) terms that take into account the

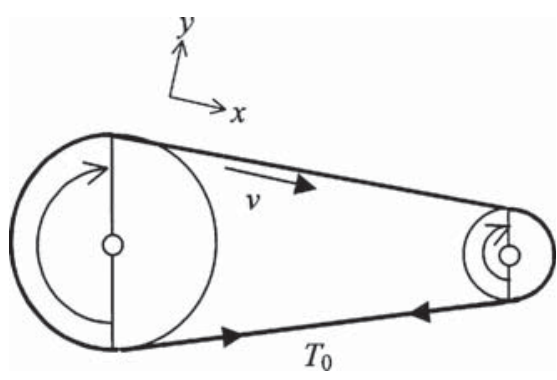

Fig. 2 Diagram of a two-pulley belt system

influence of tension in the transverse direction, Coriolis force, centrifugal force, damping, and the bending stiffness of the string. Table 1 provides a list of the dependent and independent variables of equations (1) and (2).

A test rig was constructed that consisted of a two-pulley belt-based power transmission system (see Fig. 3). The initial tension $\left(T_{0}\right)$ of the belt in the rig was adjustable via a screw/slide mechanism that altered the distance between the driven and driving pulleys. Belt tension was measured through the application of a model relating belt span resonance frequency to belt tension as defined in equation (2). The value of the mass per unit length of the belt was obtained from manufacturers data. Belt vibration data were acquired from the belt under the following six conditions: $T_{0}=98,138,198,255,315$, and $374 \mathrm{~N}$. Vibration data sets were acquired from the centre of a span of the belt when the belt was travelling at $3 \mathrm{~m} / \mathrm{s}$.

In order to measure the magnitude of belt transverse vibration an infrared-based vibration sensor was applied. This sensor converted the magnitude of the distance of a vibrating belt from the sensor by measuring the level of non-coherent infrared light reflected from the surface of the belt. The source of the infrared light is contained within the transducer and consists of a light emitting diode whose output is modulated in order to minimize the effect of background infrared radiation on the vibration measurement. The level of the reflected infrared radiation is measured by the sensor through the application of a photodiode that is sensitive to light within the frequency range of the light emitted by the emitting

Table 1 The list of dependent and independent variables

\begin{tabular}{lll}
\hline$t$ & Time & seconds \\
$T_{0}$ & Initial tension & Newtons \\
$y$ & Horizontal position within a two-dimensional Cartesian coordinate system & metres \\
$x$ & Vertical position within a two-dimensional Cartesian coordinate system & metres \\
$m$ & Mass per unit length & $\mathrm{kilograms} / \mathrm{metre}$ \\
$\Gamma$ & Damping coefficient & $\mathrm{metres} / \mathrm{second}$ \\
$v$ & String velocity & $\mathrm{metres} / \mathrm{second}$ \\
$c$ & Wave velocity & $\mathrm{m}$ \\
\hline
\end{tabular}




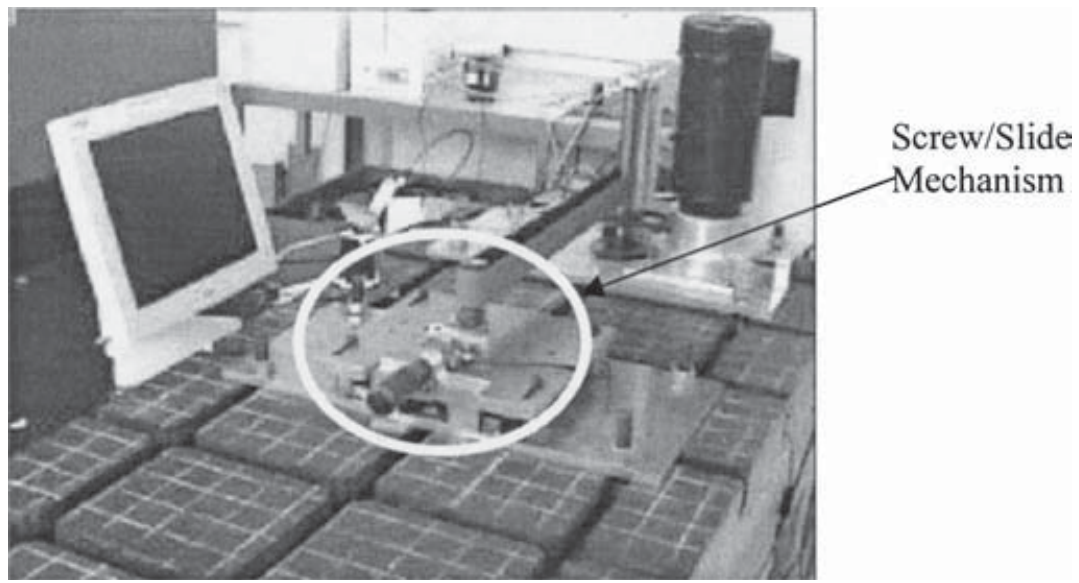

Fig. 3 The test rig mimicking a typical mail belt drive with its mechanism for tension adjustment shown inside the ellipse

diode. The sensor is sensitive to belt vibration within the frequency range of $10-100 \mathrm{~Hz}$. Table 2 provides typical measured values for the resonance frequency of the belt. These frequencies were measured in a stationary belt span. They were acquired from a belt span in which the resonance frequency of the span was excited through the application of an approximation of a unit impulse. The unit impulse approximation was generated by tapping the belt with an object according to manufacturers guidelines. The output of the vibration transducer was converted to a digital signal through the application of an analogue-to-digital (A/D) converter with a maximum sampling rate of $500 \mathrm{~Hz}$ and a resolution of 12 bits.

Figure 4 is a plot of the transverse motion of the centre of a moving belt span measured with the optical vibration sensor. The belt velocity was $3 \mathrm{~m} / \mathrm{s}$. Further information regarding the belt is given later in Table 4. Figure 4 consists of a plot of a $3 \mathrm{~s}$ segment of vibration from an element of the set of vibration data processed to train the novelty detector as described in section 3. Apparent from visual inspection of the signal is the fact that the signal is amplitude modulated and consists of multiple sinusoidal components of significant amplitude. A quantitative analysis of the characteristics of such belt vibration signals may be found in reference [2].

Table 2 Typical resonance frequencies for a stationary belt

\begin{tabular}{ll}
\hline Tension & Resonance frequency $(\mathrm{Hz})$ \\
\hline 375 & 20.2 \\
314 & 18.6 \\
255 & 16.8 \\
198 & 14.8 \\
138 & 12.4 \\
90 & 10 \\
\hline
\end{tabular}

\subsection{Sensitivity of belt vibration to changes in tension}

If certain characteristics of the transverse vibration of a moving belt are sensitive to changes in the initial tension, then by only monitoring changes in those characteristics it may be possible to detect changes in tension indirectly. However, currently most of these characteristics are either unknown or not sufficiently understood. Although the resonance frequency of a belt span is known to be dependent on the tension of the span, monitoring tension in a belt span using vibration requires the excitation of vibration in the belt span at the resonance frequency. This is the case for both stationary and moving belts, but in the case of a moving belt, vibration of a frequency other than the resonance frequency of a belt span may be present also, such as the rotational frequency of the system pulleys. Therefore, novelty detection (ND) is used in the absence of such knowledge in order to establish whether the belt vibration signal itself is sensitive to changes in the initial belt tension. The ND-based approach circumvents the need to introduce a source of excitation other than that which occurs in the system during the operation of the belt and can hence reduce the interference with belt drives during the execution of condition-based maintenance. The next section demonstrates how this may be achieved.

\section{NOVELTY DETECTION TO IDENTIFY TENSION CHANGES IN MOVING BELTS}

In this section ND is applied in order to explore the feasibility of the notion of detecting a change in initial tension by obtaining and processing the 


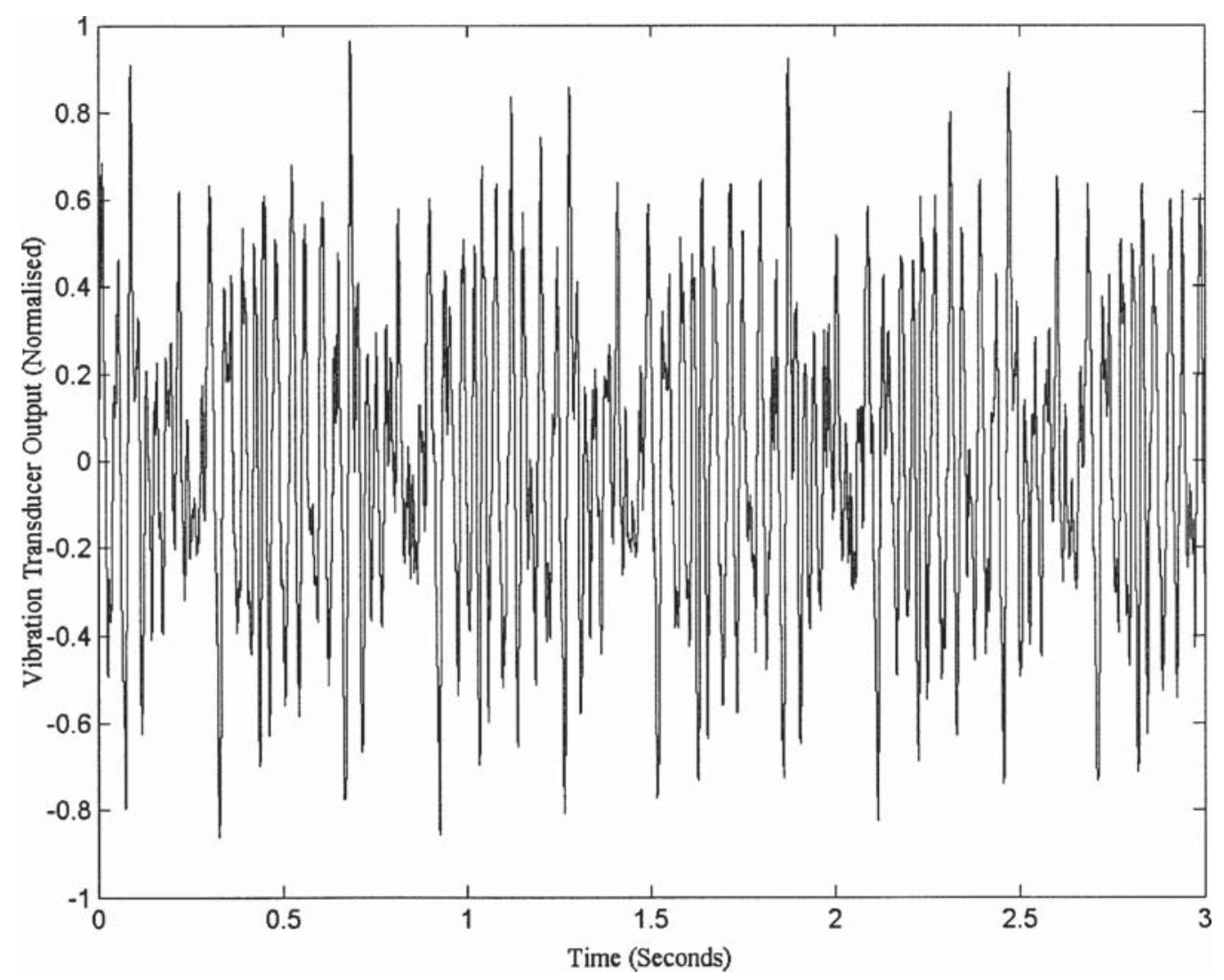

Fig. 4 Plot of a segment of data from an element of the set of vibration data with an initial belt tension of $90 \mathrm{~N}$

transverse vibration of a moving belt span without applying a unit impulse to the span.

\subsection{Novelty detection and its applicability for process control in general}

In recent years neural networks (NNs) have been increasingly used to classify the present process states into various known normal or abnormal conditions. The abnormal conditions that NNs are trained to recognize are pre-specified and in limited numbers. Most NNs require examples from more than one class in order to group their results into one of those classes. However, one major difficulty with such NNs is that it is impossible to know all the possible classes of abnormality $a$ priori. Processes and systems can exhibit abnormal conditions that have never occurred before. However, it is still important that any abnormal condition is detected quickly. Novelty detection has been receiving increasing attention as an effective method of detecting abnormal conditions in process control and condition monitoring applications (e.g. see references [17] and [18]). This is because ND is able to identify a great range of abnormal conditions without ever requiring any a priori training examples other than those associated with the commonly found and easily available examples from normal conditions. ND provides a practical solution in the face of the unpredictability and the sheer size of the possible number of variations for abnormal events.

In essence, ND is able to create models of a labelled subspace (training class or data) within a given problem space. Any new data from the problem space is compared against the ND model to check its similarity or difference, which ultimately results in the new data being classed as either 'novel' (meaning not from the training subspace) or 'non-novel' (meaning from the same subspace as the training data). References [19] and [20] provide extensive reviews of the techniques for implementing ND in general.

\subsection{The experimental set-up and the results}

The vibration data associated with a given tension at normal operating conditions for a specific type of belt while in motion (i.e. at a given length and speed) was used to train various ND models. Several factors were taken into consideration when selecting the initial sampling rate. These factors include the Nyquist criterion, the roll-off rate of the low-pass 
Table 3 The results of testing the trained ND with 7000 example windows at different window sizes and at various belt tension $\left(T_{0}\right)$ values for the same belt

\begin{tabular}{|c|c|c|c|c|c|c|}
\hline \multicolumn{2}{|c|}{ ND parameters } & \multicolumn{5}{|c|}{ Detection accuracy of ND (\%) } \\
\hline Window size & Desired sampling rate $(\mathrm{Hz})$ & $T_{0}=138$ & $T_{0}=198$ & $T_{0}=255$ & $T_{0}=315$ & $T_{0}=374$ \\
\hline 10 & 22.14 & 6.41 & 3.35 & 1.86 & 11.07 & 12.87 \\
\hline 20 & 44.29 & 10.01 & 18.751 & 7.8 & 65.76 & 67.60 \\
\hline 35 & 77.50 & 75.00 & 74.55 & 64.70 & 100 & 100 \\
\hline 45 & 99.64 & 58.00 & 70.00 & 62.4 & 100 & 100 \\
\hline $45^{*}$ & 99.64 & 82.08 & 89.00 & 89.58 & 100 & 100 \\
\hline 55 & 121.79 & 99.75 & 98.71 & 99.88 & 100 & 100 \\
\hline
\end{tabular}

$*$ Number of training examples $=5000$.

element of the frequency response of the vibration transducer, and data storage requirements. However, since different window sizes were applied to the initial samples the desired sampling rates were recalculated based on each window size. The vibration signals sampled at various frequencies were presented as time-series windows for input to NDs that were implemented as Gaussian mixture models (e.g. see pages 79 and 168 of reference [21]). The training windows thus created from belt vibration data at a constant tension of $90 \mathrm{~N}$ were used to create probability models to represent the normal initial tension of a moving belt. Vibration data related to six different tension settings (i.e. 90, 138, 198, 255, 315 , and $374 \mathrm{~N}$ ) for the same belt were used to test the trained ND models (see Table 3). Figure 5 shows examples of time-series windows for signals collected at tensions of 90 and $374 \mathrm{~N}$ respectively, where the window for $374 \mathrm{~N}$ represents a non-negligible change in the normal belt tension of $90 \mathrm{~N}$. Details of the training procedure used and available methods may be found in reference [22]. Table 4 describes the training parameters.
Table 4 Training parameters for novelty detection

\begin{tabular}{ll}
\hline Belt length & $1.4 \mathrm{~m}$ \\
Belt speed & $3 \mathrm{~m} / \mathrm{s}$ \\
Normal belt tension & $90 \mathrm{~N}$ \\
Training examples & 3000 \\
Gaussian centres & 5 \\
Type I error (=100 - accuracy on previously unseen & $0.3 \%$ \\
$\quad$ normal data) & \\
\hline
\end{tabular}

In these experiments a type I error refers to a detection error occurring when the vibration signals are obtained from the belt at the same initial belt tension value as that with which ND was trained, i.e. at $T_{0}=90 \mathrm{~N}$. When signals are related to $T_{0}=90 \mathrm{~N}$ and are mistakenly rejected as if being associated with belt tension at significantly different $T_{0}$ values then a type I error has occurred. ND thresholds were set so that the type I error on all the ND models were closely similar. In this way, all the models were shown to provide approximately 99.7 per cent accuracy when tested with previously unseen belt vibration signals at an initial belt tension of $90 \mathrm{~N}$. This arrangement provided a sound basis for comparing the

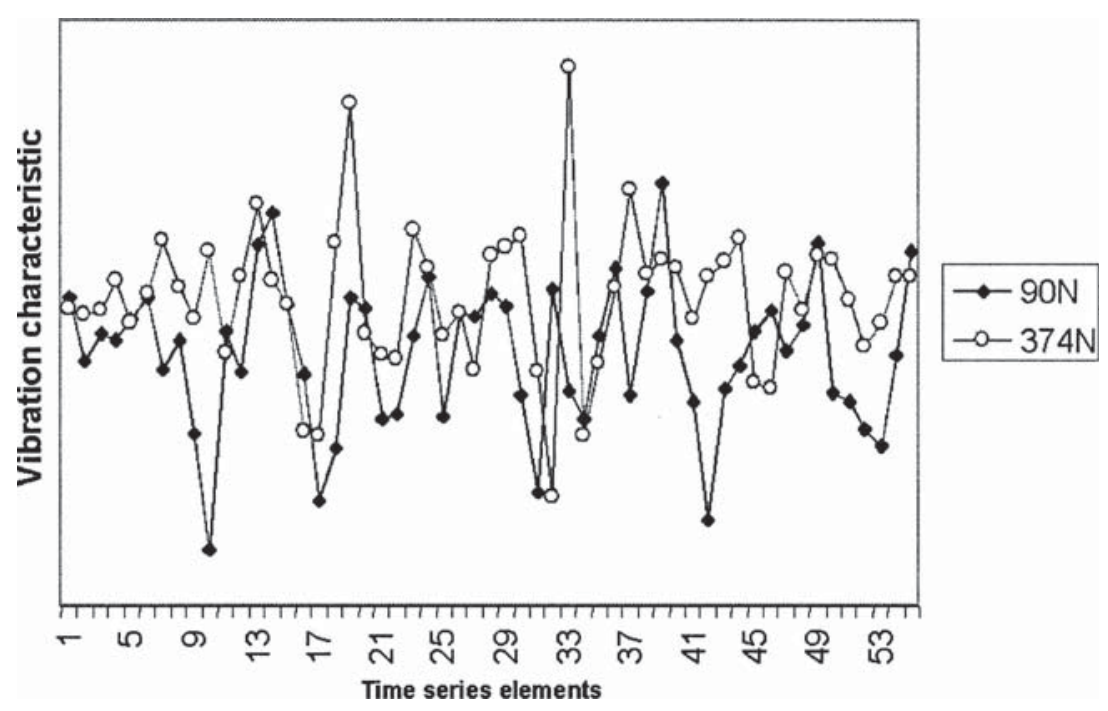

Fig. 5 Examples of input windows tested by novelty detection 
accuracy performance of different models. Any input window that plotted at or below the threshold was regarded as 'novel' (i.e. abnormal) and otherwise as non-novel or normal.

As shown in Table 3, increasing the input window size resulted in an overall improvement on accuracy performance in detection of tension changes. This was especially true when the amount of tension change was high. The window size of 55 provided the best accuracy results (average 99.67 per cent for both normal and abnormal conditions). The associated sampling rate was at $121.79 \mathrm{~Hz}$.

The experiments proved that ND was capable of providing high accuracy to detect changes in the belt tension for the moving belt without applying any unit impulse to the span. The ideas for an on-line indirect sensing are embodied in the system outlined in section 4 .

\section{INTEGRATED SYSTEM FOR AN INTERNET- BASED CONDITION MONITORING}

The proposed system, as outlined in Fig. 6, is an internet-based condition monitoring (e-CM) prototype that has successfully identified abnormal tension in moving belts. The signal obtained from an optical sensor describes the vibration of belts used for transporting letters in automated mail sorting machines. It is shown that tension changes in moving belts can

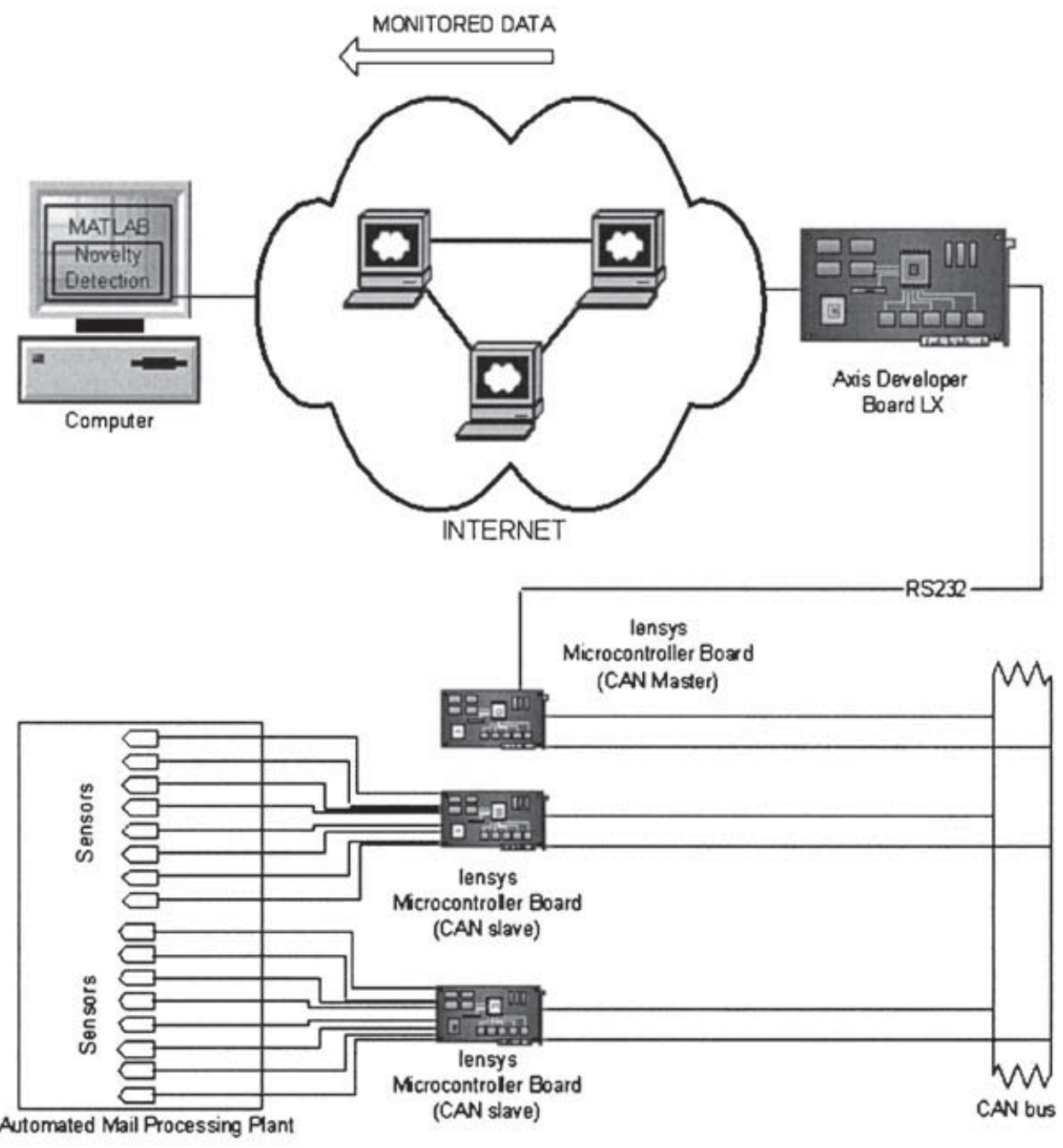

Fig. 6 A schematic diagram of the internet-based condition monitoring system proposed for implementation in automated mail processing plants 
be detected without needing to apply the existing techniques that require exciting the belts to their natural frequencies. Thus, unlike the existing techniques, which interfere with the monitored moving belt, the proposed approach allows the belts to remain in motion. This is obtained by applying ND to decide the status of belt tension by examining the signal from an optical sensor. As for linking the system components, processors, data acquisition boards, and sensors, a novel method for industrial network communication that uses XML to create a single standard format for sensor information is used. This arrangement allows sensors to be linked with the process controller via the internet. Again, this is in contrast to the dominantly non-standardized and complicated local communication and control strategies. It also requires much less cabling to connect sensors to the personal computer that runs the ND software. Finally, in order to reduce costs further, a new general-purpose low-cost microcontroller board with a built-in CAN capability is used for data acquisition, while another low-cost microcontroller board with a built-in ethernet link is used for direct transmission through the internet.

The main components of the system include the following.

1. Sensors for monitoring the tension in the belts.

2. Microcontroller boards for acquiring and preprocessing sensor values.

3. A microcontroller board for connection to the internet.

4. A PC pre-loaded with MATLAB software, a software implementation of the novelty detection algorithm, and connection to the internet.

The microcontroller board, model A030601, by Intelligent Engineering Systems (IENSYS) Limited $\mathrm{UK}$, is a general-purpose board for data acquisition and control. The board is based on the Microchip [23] PIC18F458 microcontroller. The microcontroller has up to eight analogue input channels and a 10-bit resolution A/D converter. The board which has 34 digital input/output (I/O) pins also includes RS232 and CAN support, which complies with the ISO CAN conformance test. The microcontroller can be programmed using a microchip in-circuit debugger (ICD2). The development environment is microchip MPLAB [23] and the programs can be written in Assembly or $\mathrm{C}$ language. The board also has a Dallas $^{\circledR}$ one-wire bus and an embedded one-wire Dallas DS2438Z [24] battery monitoring chip. The board uses this chip to monitor its temperature, d.c. input voltage, and the regulated d.c. voltage for self-diagnosis purposes.
The other microcontroller board, called the axis developer board LX by Axis Communications Sweden, is used for internet connectivity with an embedded Linux operating system. The board comprises two RS232, two parallel, and one RS485 interface and comes with a webserver that supports a common gateway interface (CGI) for incorporating dynamic content.

The MATLAB program sends the request for the sensor data through the internet. A CGI program written in $\mathrm{C}$ in the developer board receives this request and gets the sensor data from the CAN master, which collects the sensor data from the CAN slaves via RS232. Once all the sensor data (see Fig. 7) is updated it is incorporated in an XML document (see Fig. 8) by the CGI program. XML is used to describe the features of a sensor, which is impossible to do with HTML. An advantage of the system is that even if the system is switched to some other industrial network, the same XML document will be created. This means that the only change that will be needed will be at the data acquisition part, while the remaining software and hardware will remain the same.

Once ready, the XML document is then sent to the MATLAB program to be parsed so that the sensor data is extracted and fed to the ND algorithm for further processing (as described in section 3.2). The program flowchart for the CGI program is shown in Fig. 9 and the program code written to implement the last box in the flowchart is given in Fig. 10.

\begin{tabular}{|c|c|}
\hline \multirow{4}{*}{ SENSOR } & IDENTIFICATION \\
\cline { 2 - 2 } & TYPE \\
\cline { 2 - 2 } & INSTALLED_LOCATION \\
\cline { 2 - 2 } & MEASURED_VALUE \\
\cline { 2 - 2 } & REQUIRED_VALUE \\
\cline { 2 - 2 } & MIN_ALLOWED_VALUE \\
\cline { 2 - 2 } & MAX_ALLOWED_VALUE \\
\cline { 2 - 2 } & ACTUATOR \\
\hline
\end{tabular}

Fig. 7 A basic representation of a sensor and its features

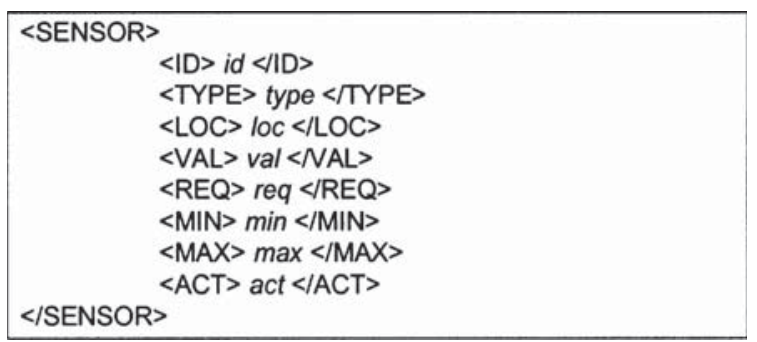

Fig. 8 XML tags for the basic representation of a sensor 


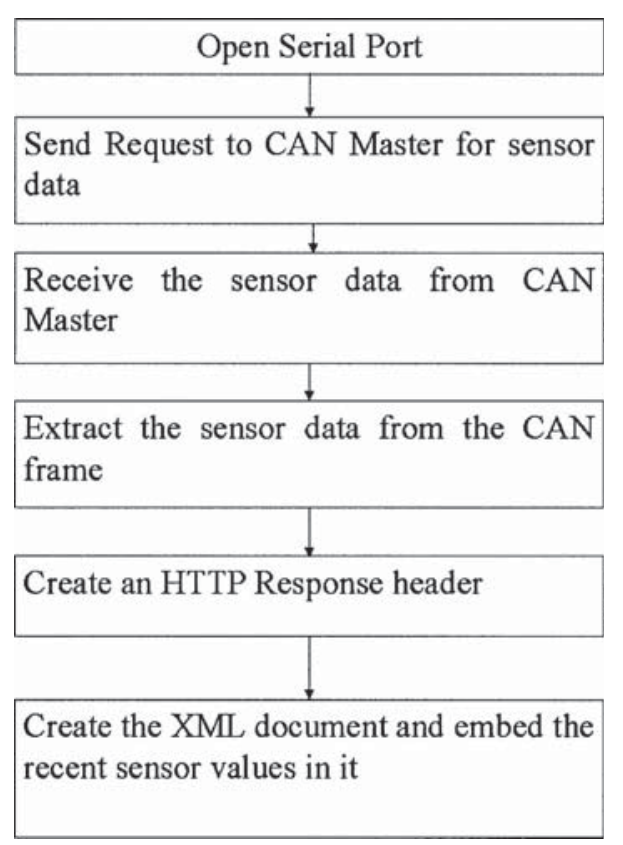

Fig. 9 The flowchart for the XML document generator

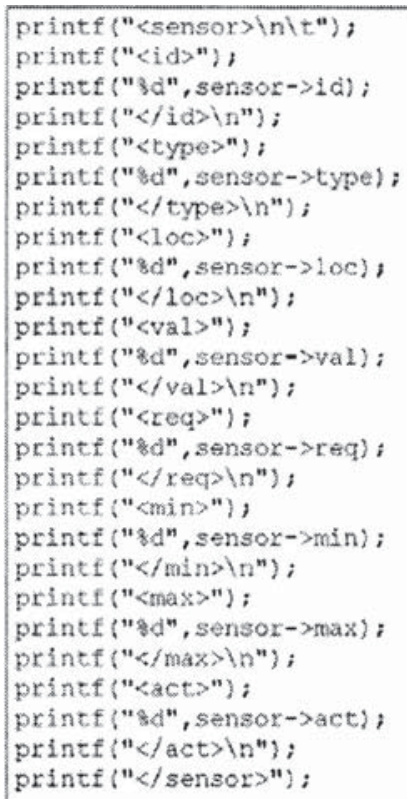

Fig. 10 Using the C code an XML document is created each time the client requests the sensor data. Instead of the variables sensor- $>$ val, etc., the recent value of the sensor is embedded and sent to the client

If the request is sent by a web browser then the response on the web browser will be similar to the snapshot shown in Fig. 8. The only difference would be that the variable shown in italics in Fig. 8 will be replaced by actual recent values.

\section{DISCUSSION}

\subsection{XML and e-CM}

Numerous industrial networks can be used to implement automated condition monitoring and control, e.g. CAN, Profibus, etc. Unfortunately, all these networks have different protocols, and hence they cannot communicate with each other. There is a growing realization in industry that there must be an open standard that can enable interoperability between different industrial networks. The solutions already available in the market provide limited functionality and support. XML is used here as a solution for the integration of information between various industrial networks, especially for e-CM. With this approach sensor data from different industrial networks can be converted into a single standard XML document in order to define sensors and the information that they provide.

A very visible shift in the industry is that more and more people are moving towards open standards. These standards are preferred as their specifications are freely available to anyone interested in their implementation. Open standards used in this project include TCP/IP, Linux, and XML.

Ethernet and internet are building inroads into the field of condition monitoring and control because of various advantages, such as low implementation costs, ease of use, worldwide application, and openness. An added advantage with e-CM is that data from spatially and/or geographically dispersed locations can be accessed, analysed, and compared at a single location. This increases data fusion capabilities to a great extent.

e-CM has been found to be extremely effective in situations where a system operates with a known fault or may operate beyond its original design limitations. It is most effective in situations where a system has to be remotely accessed. It must, however, be made certain that the e-CM system is responsive enough so that a clear idea about the condition of the system can be made.

The future architecture of the internet, presented on the website of the World Wide Web Consortium (W3C), is based on XML. W3C is a group of around 400 member organizations from all over the globe controlling the development of the internet. XML is extremely suitable for exchanging data across the internet and elsewhere. It is easy to learn and implement yet is powerful enough to handle even the most complex of applications. XML fulfils the goals of openness and hence interoperability. Linux is also becoming popular because of its openness and 
stability. The use of Linux OS is steadily increasing, especially in embedded applications.

The immediate implication for UK's Royal Mail, with its large network of mail processing plants, is the ability to obtain automatic and continuous condition monitoring of large numbers of belts at critical or bottleneck areas within its various types of automated mail processing machinery.

\subsection{Novelty detection and feature extraction for belt $\mathbf{C M}$}

As explained in section 3.2, a time window from the vibration signal was used as input to the novelty detection model. The use of belt vibration features other than a window of time domain data as the input of a novelty detector could circumvent the need to select a specific length of time window in the following sense: the use of certain statistical features as the input to a novelty detector can result in independence between the dimensionality of the feature that forms the input to the novelty detector and the length of the data window from which extraction of the feature occurs. However, the use of statistical features as the input of a novelty detector would still result in having to undertake the process of selecting an appropriate time window, albeit at a different stage in the design cycle of a novelty detector. For example, if the chosen statistical feature consisted of a discretized Fourier decomposition of belt vibration then the frequency resolution of the decomposition would have to be selected. If the chosen feature consists of a second-order moment, higher-order moment, or indeed any other statistical feature, then it would be necessary to select the length of the data window from which to extract such a feature so that appropriate minimization of the bias/variance or, at the very least, the variance of the estimate of the feature for a particular fault mode occurs (assuming the ergodicity of the feature).

Assuming that excitation of an appropriate magnitude of the resonance mode of the belt span occurs during the acquisition of belt vibration data, then one such frequency domain feature could consist of the energy dissipated at the resonance frequency of the belt. The general criterion for identifying frequency domain features or any other characteristics/features of the belt vibration data consists of the result of evaluating the features in terms of the distance between points in the feature space when a change in tension occurs. A knowledge of the belt vibration features that maximizes or results in a significant distance between points in the feature space after a change in belt tension has occurred could facilitate the design of conceptually and computationally simpler condition classification algorithms than ND. However, such knowledge is yet to be obtained and ND has been shown to provide an effective solution in its absence.

\section{CONCLUSION}

An e-CM system capable of providing alarms when unacceptable belt tensions occur has been outlined and demonstrated. The system uses various new technologies for e-CM, such as embedded Linux OS, distributed monitoring system, novelty detection, and XML. The existing condition-based monitoring systems for belts require direct measurement relying on expensive, inflexible, and manual data collection. The application of novelty detection for processing transverse vibration of a moving belt span without exciting the natural frequency of the belt span has been proven. By smartly combining appropriate vibration sensor, vibration features, novelty detection, low-cost data acquisition, and the XML standard for communication, a cost-effective maintenance solution has been obtained. The resulting integrated approach is more efficient because: (a) it can reduce waste by minimizing process interruptions caused by direct belt inspection methods while obtaining a high detection accuracy (99.67 per cent) and (b) it can provide on-line remote $\mathrm{CM}$ that is cost-effective, simple, standardized, and scalable across a wide area and for a relatively large number of sensors. This improvement is especially important when applied to bottleneck processes and critical components.

Future work can provide graded novelty detection in order to reduce sensitivity of ND when the belt tension changes are not significant enough to pose any threat to the health of the components in the belt drive system.

If a fault mode affects characteristics of belt vibration then the occurrence of that fault mode causes a change in the characteristics of the belt vibration. Since novelty detection detects changes in characteristics of belt vibration, an appropriately trained novelty detector could detect such fault modes. This conclusion has not been tested empirically for all possible fault modes; the testing of this conclusion with respect to particular fault modes could constitute a possible future direction for this work.

Finally, during system improvement exercises it is not easy to select and use the optimum combination of the available technologies. This difficulty may explain why engineers who are looking for better 
solutions to the existing problems tend to experiment with new off-the-shelf solutions that are usually very expensive. Ever-increasing completion requires the engineers to examine several new approaches concurrently in order to address different parts of a given problem while adopting a holistic evaluation of their choices. Committed collaboration with researchers in technical universities can bear fruit towards such goals, as demonstrated by this paper.

\section{ACKNOWLEDGEMENTS}

The authors would like to thank Royal Mail Group plc and the Engineering and Physical Sciences Research Council for supporting this study.

\section{REFERENCES}

1 Notini, L. Condition monitoring of belt based motion transmission systems, PhD Thesis, Wolfson School of Mechanical and Manufacturing Engineering, Loughborough University, 2004.

2 Notini, L., Grueter, S., Zorriassatine, F., Parkin, R. M., Jackson, M. R., and Coy, J. A technique for the condition monitoring of power transmission belts. In the International Conference on Mechatonics 2003 (ICOM 2003), Loughborough University, Loughborough, 15-27 June 2003, paper 1860584209, pp. 453-458.

3 Kozhevnikov, S. N. Vibration of a two stage speed vee-belt drive, Sov. Engng Res., 1982, 2(5), 01446622, 33-34.

4 Abrate, S. Vibrations of belts and belt drives, Mech. Mach. Theory, 1992, 27(6), 0094-114X, 645-659.

5 Wang, K. W. and Mote, C. D. J. Band/wheel system vibration under impulsive boundary excitation, J. Sound Vibr., 1987, 115(2), 0022-460X, 203-216.

6 Yamashina, H., Okumara, S., and Kawai, I. Development of a diagnosis technique for failures of $\mathrm{V}$-belts by a cross-spectrum method and a discriminant function approach, J. Intell. Mtg, 1996, 7(1), 09565515, 85-93.

7 Zorriassatine, F., Al-Habaibeh, A., Notini, L., Salvan, S., Parkin, R. M., Jackson, M. R., and Coy, J. An integrated sensory system for intelligent condition monitoring of mail processing machines. In Congress on Condition Monitoring and Diagnostic Engineering Management (COMADEM 2003), Vaxjo University, Sweden, August 2003, pp. 545-554.

8 Cowan, R. S. and Winer, W. O. Diagnostic and prognostic tools for machinery health monitoring.
In Congress on Condition Monitoring and Diagnostic Engineering Management (COMADEM 2000), 2000, pp. $577-586$.

9 Hannah, P., Starr, A., and Bryanston-Cross, P. Condition monitoring and diagnostic engineering a data fusion approach. In Proceedings of the 14th International Congress on Condition Monitoring and Diagnostic Engineering Management (COMADEM 2001), Manchester, 4-6 September 2001, pp. 275-282.

10 Reichard, K. M., Van Dyke, M., and Maynard, K. Application of sensor fusion and signal classification techniques in a distributed machinery condition monitoring system. In Proceedings of SPIE Conference on Sensor Fusion: Architectures, Algorithms, and Applications, Vol. IV, Orlando, Florida, 25-28 April 2000, Vol. 4051, 2000, pp. 329-336 (The International Society for Optical Engineering).

11 Sofronas, T. Case 1: the fatigued motor shaft, Hydrocarbon Processing, 2001, 80, 85-86.

12 Swift, H. W. Power transmission by belts: an investigation of fundamentals, Proc. Instn Mech. Engrs, 1928, 2, 659.

13 Fawcett, J. N. Chains and belt drives: a review, Shock Vibr. Dig., 1981, 13, 5-12.

14 Fawcett, J. N., Burdess, J. S., and Hewit, J. R. Initial tension setting for drive belts, Automot. Engr, 1988, 13, 70-71.

15 Pellicano, F., Catellani, G., and Fregolent, A. Parametric instability of belts: theory and experiments, Computers and Structs, 2004, 82, 81-91.

16 Naguleswaran, S. and Williams, C. J. H. Lateral vibrations of band-saw blades and the like, Int. J. Mech. Sci., 1968, 10, 239-250.

17 Jack, L. B. and Nandi, A. K. Novelty detection using minimum variance features. Proceedings of the 14th International Congress on Condition Monitoring and Diagnostic Engineering Management (COMADEM 2001), Manchester, 4-6 September 2001, pp. 111-118.

18 Tarassenko, L., Nairac, A., Townsend, N., Buxton, I., and Cowley, P. Novelty detection for the identification of abnormalities, Int. J. Systems Sci., November 2000, 31(11), 1427-1439.

19 Markou, M. and Singh, S. Novelty detection: a review - Part 1: statistical approaches, Signal Processing, December 2003, 83(12), 2481-2497.

20 Markou, M. and Singh, S. Novelty detection: a review - Part 2: neural network based approaches, Signal Processing, December 2003, 83(12), 2499-2521.

21 Nabney, I. T. NETLAB: algorithms for pattern recognition, 2002 (Springer, London).

22 Zorriassatine, F., O'Brien, C., and Tannock, J. D. T. Using novelty detection to identify abnormalities caused by mean shifts in bivariate processes. Computers and Ind. Engng, March 2003, 44(3), 385-408.

23 http://www.microchip.com.

24 http://pdfserv.maxim-ic.com/arpdf/DS2438.pdf. 